\title{
Effect of short-term blood pressure variability on functional outcome after intra-arterial treatment in acute stroke patients with large-vessel occlusion
}

Tianli Zhang, Xiaolong Wang, Chao Wen, Feng Zhou, Shengwei Gao, Xiaodong Zhang, Shiqin Lin, Jing Shi and Weirong Li*iD

\begin{abstract}
Background: Endovascular treatment (EVT) is advocated for acute ischaemic stroke with large-vessel occlusion $(L V O)$, but perioperative periods are challenging. This study investigated the relationship between post-EVT shortterm blood pressure variability (BPV) and early outcomes in LVO patients.

Methods: We retrospectively reviewed 72 LVO patients undergoing EVT between June 2015 and June 2018. Hourly systolic and diastolic blood pressures (SBP and DBP, respectively) were recorded in the first $24 \mathrm{~h}$ post-EVT. BPV were evaluated as standard deviation (SD), coefficient of variation (CV), and successive variation (SV) separately for SBP and DBP. Functional independence at 3 months was defined as a modified Rankin Scale (mRS) score of 0-2.

Results: For $58.3 \%$ patients with favorable outcomes, the median National Institutes of Health Stroke Scale and Alberta Stroke Program Early CT scores on admission were 14 and 8, respectively. The maximum SBP ([154.3 \pm 16.8$]$ vs. [163.5 \pm 15.6$], P=0.02$ ), systolic CV ([8. $8 \% \pm 2.0 \%]$ vs. [11.0\% \pm 1.8$], P<0.001)$, SV ([11.4 \pm 2.3$]$ vs. $[14.6 \pm 2.0], P<$ $0.001)$, and SD ([10.5 \pm 2.4$]$ vs. [13.8 \pm 3.9$], P<0.001)$ were lower in patients with favorable outcomes. On multivariable logistic regression analysis, systolic SV (OR: $4.273,95 \% \mathrm{Cl}: 1.030$ to $17.727, P=0.045$ ) independently predicted unfavorable prognosis. The area under the curve was 0.868 ( $95 \% \mathrm{Cl}: 0.781$ to $0.955, P<0.001)$, and sensitivity and specificity were $93.3 \%$ and $73.8 \%$, respectively, showing excellent predictive value for 3-month pooroutcomes.
\end{abstract}

Conclusions: Decreased systolic SV following intra-arterial therapies result in favorable outcomes at 3 months. Systolic SV may be a novel predictor of functional prognosis in LVO patients.

Keywords: Blood pressure, Acute ischemic stroke, Large vessel occlusion, Functional outcome

\section{Background}

Early endovascular treatment (EVT) for patients who had acute ischaemic stroke with large-vessel occlusion (AIS-LVO) is highly recommended based on the findings of six randomized controlled clinical trials [1-6]. However, several factors during the perioperative period of EVT, including blood pressure (BP) management, need urgent attention. The optimal range of BP following EVT remains unclear. The 2018 American Heart

\footnotetext{
* Correspondence: tyszxyysci@163.com

Department of Neurology, Taiyuan Central Hospital, Taiyuan city 030009, Shanxi Province, China
}

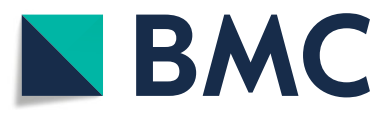

(c) The Author(s). 2019 Open Access This article is distributed under the terms of the Creative Commons Attribution 4.0 International License (http://creativecommons.org/licenses/by/4.0/), which permits unrestricted use, distribution, and reproduction in any medium, provided you give appropriate credit to the original author(s) and the source, provide a link to the Creative Commons license, and indicate if changes were made. The Creative Commons Public Domain Dedication waiver (http://creativecommons.org/publicdomain/zero/1.0/) applies to the data made available in this article, unless otherwise stated.

Association and American Stroke Association guidelines for the early management of patients with AIS recommends maintaining the BP at $<180 / 105 \mathrm{mmHg}$ (IIb, B-NR) in patients who underwent mechanical thrombectomy (MT) with successful reperfusion [7]. The 2018 Chinese guidelines also recommend a target BP of 140/90 or a $\mathrm{BP}$ of $20 \mathrm{mmHg}$ lower than that at baseline, but it should also not be less than $100 / 60 \mathrm{mmHg}$ (II, C) [8]. However, reperfusion injury may occur dispite maintaining the $\mathrm{BP}$ within the target range.

Blood pressure variability (BPV) is the fluctuation of BP over a certain period of time. In the acute stage of 
cerebrovascular disease, the fluctuation of cerebral perfusion pressure is aggravated by short-term BPV due to impaired automatic regulation of cerebral blood flow [9]. Hypertension during the perioperative period may lead to adverse events such as the reperfusion syndrome and cardiovascular complications, while hypotension may lead to hypoperfusion and increases the risk of infarction. A recent systematic review reported that increased BPV after stroke is associated with higher rates of intracranial haemorrhage and disability [10]. However, there is limited epidemiological evidence to evaluate the relationship between BP level and early functional prognosis after EVT. Thus, this study aimed to explore the association between short-term BPV in the first $24 \mathrm{~h}$ following EVT and functional outcomes in patients with AISLVO.

\section{Methods}

\section{Patient selection}

This is a retrospective study was approved by the Institutional Review Board of Taiyuan Central Hospital, Shanxi, People's Republic of China. Consecutive AIS-LVO patients who underwent emergency EVT in the tertiary care stroke center of Taiyuan Central Hospital between June 2015 and June 2018 were enrolled. The inclusion criteria were as follows: (1) age of $\geq 18$ years; (2) AIS confirmed via head computed tomography (CT) or magnetic resonance imaging at admission; (3) occlusion of the internal carotid artery or M1 of the middle cerebral artery diagnosed within $6 \mathrm{~h}$ after onset by digital subtraction angiography; (4) preoperative Alberta Stroke Program Early CT Score (ASPECTS) of $\geq 6$, prestroke modified Rankin Scale (mRS) score of $<2$, and National Institutes of Health Stroke Scale (NIHSS) score of $\geq 6$; (5) treatment initiated (groin puncture) within $6 \mathrm{~h}$ of symptom onset; (6) clinical features and BP recorded at baseline and hourly for at least $24 \mathrm{~h}$ after EVT; and (7) follow up by phone or face-to-face consultations at 3 months with complete documentation. Patients were excluded if they had active bleeding or a bleeding tendency (including primary intracerebral haemorrhage, and potential causes such as gastrointestinal malignancy, liver cirrhosis, renal failure, hematologic tumour, vitamin $\mathrm{K}$ deficiency, and sepsis, which could lead to bleeding events), serious heart failure or respiratory failure pre-admission, glucose $<50 \mathrm{mg} / \mathrm{dL}$ or $>400 \mathrm{mg} / \mathrm{dL}$, severe hypertension beyond drug control, and severe non-cardiovascular events that occurred within 3 months of follow-up. The management of patients with AIS-LVO was based on the Chinese guidelines for diagnosis and treatment of AIS 2014 and the Chinese guidelines for the endovascular treatment of acute ischemic stroke 2015 [11, 12].

\section{Data collection}

Baseline characteristics such as demographics, vascular risk factors, previous use of anti-platelet aggregation drugs, Trial of ORG 10172 in acute stroke treatment (TOAST) types on admission, NIHSS scores on admission, ASPECTS on admission, systolic BP (SBP) and diastolic $\mathrm{BP}$ (DBP) on admission, laboratory values, and type of treatment for the EVT were collected. The degree of recanalization at the end of EVT was measured using the Thrombolysis in Cerebral Infarction (TICI) score [13] as obtained from the reports of interventional specialists (C.W. and F.Z.). All patients were examined via brain $\mathrm{CT}$ in the first $24 \mathrm{~h}$ after EVT to determine any changes in intracranial haemorrhage using the criteria developed by the European Cooperative Acute Stroke Study (ECASS) [14]: HI1, small petechiae with an indistinct border within the vascular territory; HI2, more confluent petechiae, no mass effect; PHI, hematoma within infarcted tissue, occupying $<30 \%$ of the infarcted area, no substantive mass effect; and $\mathrm{PH} 2,>30 \%$ of the infarcted area with significant space-occupying effect or parenchymal hematoma distant from the infarcted brain tissue.

\section{BP monitoring and BPV presentation post EVT}

The hourly SBP and DBP of all patients were recorded during the first $24 \mathrm{~h}$ following EVT. Postoperative management of blood pressure depended on whether the responsible vessels were successfully recanalized according to the Chinese guidelines for the endovascular treatment of acute ischaemic stroke 2015 [12], which recommend a target $\mathrm{BP}$ of $20-30 \mathrm{mmHg}$ lower than that at baseline, but it should not be less than $90 / 60 \mathrm{mmHg}$ in patients with successful recanalization. For patients without successful recanalization, permissive hypertension was set at a systolic blood pressure more than 150 but not exceeding $180 \mathrm{mmHg}$. For patients pretreated with intravenous thrombolysis, permissive hypertension was set at $<180 /$ $105 \mathrm{mmHg}$. All patients with postoperative hypertension were treated with intravenous urapidil (first choice) or sodium nitroprusside (second choice) when BP levels exceeded the former prespecified cut-offs. We documented the maximum, minimum, and mean arterial BP (MAP, [SBP $+2 \times \mathrm{DBP}] / 3$ ) levels for each individual. Based on previously published studies, BPV was calculated using the following equation:

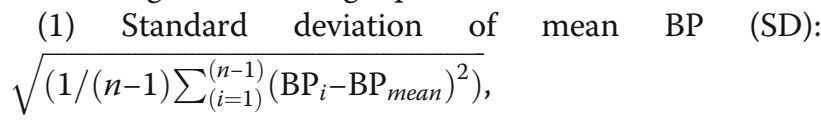

(2) Coefficient of variability (CV [\%]): $\mathrm{SD} / \mathrm{BP}_{\text {mean }} \times$ 100 ,

$$
\begin{array}{ll}
\text { Successive } & \text { variation } \\
\sqrt{\left(1 /(n-1) \sum_{(i=1)}^{(n-1)}\left(\mathrm{BP}_{i+1}-\mathrm{BP}_{i}\right)^{2}\right)} & {[15] .}
\end{array}
$$




\section{Evaluation of functional prognosis}

Functional outcome was evaluated at 3 months by certified neurologists using the mRS score. The patients were then divided into two groups based on the functional outcome score: the favorable and unfavorable outcome groups comprised patients with mRS 0-2 and mRS 3-5, respectively. The mRS scores were determined based on the follow-up findings.

\section{Statistical analysis}

All data analyses were performed using the SPSS V. 25.0 software. Continuous variables were expressed as means \pm $\mathrm{SD}$ (normal distribution) or median with interquartile range (IQR) (skewed distribution). Comparisons between groups were conducted using the Students t-test, MannWhitney $U$ test, or $\chi^{2}$ test, or One-way ANOVA analysis as appropriate. Univariable and multivariable logistic regression models were used to explore the association between BPV indices during the first $24 \mathrm{~h}$ post EVT with 3-month functional outcome before and after adjustment for the following potential confounders: age, sex, hypertension, coronary heart disease, atrial fibrillation, diabetes mellitus, smoking, admission NIHSS scores, admission serum glucose and LDL-C levels, admission SBP and DBP levels, type of anesthesia (general anesthesia vs conscious sedation), baseline ASPECTS, onset to groin puncture time, vascular lesion (M1 of the middle cerebral artery [MCA] vs ICA), frequency of MT, type of EVT and rates of successful recanalization. In the initial univariable analyses, a $P$ value $<0.05$ was set as the threshold for inclusion in the multivariable models. Odds ratios (OR) and $95 \%$ confidence interval $(\mathrm{CI})$ were calculated to determine any associations.

To determine the predictive capabilities according to SBP SV, the receiver operating characteristic (ROC) curves were generated, and the sensitivities, specificities, positive predictive values (PPV) and negative predictive values (NPV) of systolic SV were calculated. Because the interaction between BPV and successful reperfusion or offending artery was significant, a subgroup analysis by BPV parameters with 3-month functional outcome were used. We also examined the impact of BPV on functional outcome based on different systolic SV. Patients were stratified according to the quartile of their systolic SV during the first $24 \mathrm{~h}$ post EVT and the distribution of the patients with favorable outcomes was calculated in each group.

\section{Results}

\section{Patient demographics and clinical characteristics}

Among 83 patients who underwent emergency EVT in our stroke unit, 11 (13.3\%) patients were excluded owing to the following causes: $2(2.4 \%)$ died as a result of noncardiovascular disease, 4 (4.8\%) had inadequate BP during the first $24 \mathrm{~h}, 3$ (3.6\%) exited the study during the 3-month follow-up, and $2(2.4 \%)$ died as a result of cerebral hernia. As a result, 72 patients with AIS-LVO within the anterior circulation were enrolled in this study.

The baseline clinical demographic characteristics of the study population are summarized in Table 1 . Of the 72 patients, including $42(58.3 \%)$ with favorable outcomes and $30(41.7 \%)$ with unfavorable outcomes at 3months, the mean age was $64.8 \pm 10.9$ years, and 27 $(37.5 \%)$ were women. The median NIHSS score at admission was 14 points [IQR, 9-19], while the median ASPECTS was 8 points [IQR, 7-9]. Of the 72 patients, $86.1 \%$ patients achieved recanalization (TICI $2 \mathrm{~b}$ or 3 ). In total, $26.4 \%$ patients received combined intravenous thrombolysis and thrombectomy, $13.9 \%$ of patients were treated with intra-arterial thrombolysis alone, and 59.7\% of patients were treated with direct mechanical thrombectomy. Intracranial haemorrhagic transformation occurred in 13 patients (18.0\%), while the hemorrhagic transformation was no different between the patients with the three treatments of EVT (Additional file 1).

Compared to patients with an unfavorable outcome group, the NIHSS scores, admission SBP level, and frequency of MT were significantly lower in the favorable outcome group (all $P<0.05$ ). Patients with a 3 -month favorable outcome were more likely to have lesions in the M1 of middle cerebral artery, to have higher rates of successful recanalization, to have higher admission ASPECT scores, and to receive intra-arterial thrombolysis alone. The rates of vascular risk factors, time of symptom onset to groin puncture, and HI were not significantly different between the two groups.

Table 2 lists the baseline characteristics of patients in different groups, in which patients are divided into four groups according to systolic SV values quartiles: Systolic SV values $\leq 10.96,10.97-12.71,12.72-14.24$, and $>14.24$. The frequency of MT, ASPECT score at admission and rates of successful recanalization post EVT differed among the four groups (all $P<0.05$ ).

\section{BPV and 3-month functional outcome}

In this study (Fig. 1), we detected the difference in maximum SBP, systolic CV, SV, and SD between the two outcome groups. Patients with unfavorable prognosis had higher maximum SBP $([163.5 \pm 15.6]$ vs. $[154.3 \pm$ $16.8], P=0.02)$, systolic $\mathrm{CV}([11.0 \% \pm 1.8 \%]$ vs. $[8.8 \% \pm$ $2.0 \%], P<0.001), \mathrm{SV}([14.6 \pm 2.0]$ vs. $[11.4 \pm 2.3], P<$ $0.001)$, and SD ([13.8 \pm 3.9$]$ vs. [10. $5 \pm 2.4], P<0.001)$. We found no significant difference in the level of MAP, mean SBP, minimum SBP, and dates of DBP variability between the two groups $(P>0.05)$. On subgroup analysis, we also found the maximum SBP, systolic SV, CV, 
Table 1 Baseline characteristics of patients in the two outcome groups

\begin{tabular}{|c|c|c|c|c|}
\hline Variable & Total & $\begin{array}{l}\text { Favorable outcome } \\
\text { group }(n=42,58.3 \%)\end{array}$ & $\begin{array}{l}\text { Unfavorable outcome } \\
\text { group }(n=30,41.7 \%)\end{array}$ & $P$ value \\
\hline Age (years), mean $\pm S D$ & $64.8 \pm 10.9$ & $64.5 \pm 11.8$ & $65.1 \pm 9.8$ & 0.820 \\
\hline Male, n (\%) & $45(62.5)$ & $27(64.3)$ & $18(60.0)$ & 0.711 \\
\hline Hypertension, n (\%) & $48(66.7)$ & $28(66.7)$ & $20(66.7)$ & 1.000 \\
\hline Diabetes mellitus, n (\%) & $23(31.9)$ & $17(40.5)$ & $6(20.0)$ & 0.066 \\
\hline Coronary heart disease, n (\%) & $21(29.2)$ & $12(28.6)$ & $9(30.0)$ & 0.895 \\
\hline Atrial fibrillation, $\mathrm{n}(\%)$ & $24(33.3)$ & $16(31.8)$ & $8(26.7)$ & 0.310 \\
\hline Previous history of cerebrovascular disease, n (\%) & $10(13.9)$ & $5(11.9)$ & $5(16.7)$ & 0.565 \\
\hline Previous antiplatelet therapy, n (\%) & $14(19.4)$ & $9(21.4)$ & $5(16.7)$ & 0.615 \\
\hline Current smoker, n (\%) & $34(47.2)$ & $21(50.0)$ & $13(43.3)$ & 0.576 \\
\hline NIHSS score at admission, median (IQR) & $14(9-19)$ & $13(8-17)$ & $17(12-20)$ & $0.015^{\mathrm{a}}$ \\
\hline Glucose level at admission (mg/dL), mean $\pm \mathrm{SD}$ & $152.3 \pm 85.0$ & $163.8 \pm 108.0$ & $135.0 \pm 30.6$ & 0.157 \\
\hline SBP level at admission $(\mathrm{mmHg})$, mean \pm SD & $153.8 \pm 23.5$ & $146.9 \pm 18.5$ & $163.5 \pm 25.5$ & $0.003^{\mathrm{a}}$ \\
\hline DBP level at admission $(\mathrm{mmHg})$, mean $\pm \mathrm{SD}$ & $85.2 \pm 13.4$ & $83.4 \pm 12.8$ & $87.6 \pm 14.0$ & 0.189 \\
\hline LDL-C at admission (mg/dL), median (IQR) & $44.73(34.97-55.71)$ & $45.18(34.43-53.15)$ & $44.01(35.19-57.60)$ & 0.541 \\
\hline \multicolumn{5}{|l|}{ TOAST type, n (\%) } \\
\hline Large artery atherosclerosis & $44(61.1)$ & $23(54.8)$ & $21(70.0)$ & \multirow[t]{4}{*}{0.442} \\
\hline Cardioembolism & $23(31.9)$ & $16(38.1)$ & $7(23.3)$ & \\
\hline Clear reason & $4(5.6)$ & $2(4.8)$ & $2(6.7)$ & \\
\hline Unknown reason & $1(1.4)$ & $1(2.4)$ & $0(0.0)$ & \\
\hline ASPECTS at admission, median (IQR) & $8(7-9)$ & $8(8-9)$ & $7(6.75-8)$ & $<0.001^{\mathrm{a}}$ \\
\hline \multicolumn{5}{|l|}{ Vascular lesion } \\
\hline M1 of the middle cerebral artery & $51(70.8)$ & $35(83.3)$ & $16(53.3)$ & \multirow[t]{2}{*}{$0.006^{\mathrm{a}}$} \\
\hline Internal carotid artery & $21(29.2)$ & $7(16.7)$ & $14(46.7)$ & \\
\hline \multicolumn{5}{|l|}{ Type of anesthesia } \\
\hline General anesthesia, n (\%) & $9(12.5)$ & $4(9.5)$ & $5(16.7)$ & 0.366 \\
\hline Conscious sedation, n (\%) & $63(87.5)$ & $38(90.5)$ & $25(83.3)$ & 0.366 \\
\hline Time from stroke onset to groin puncture $(\mathrm{min})$, mean \pm SD & $290.5 \pm 80.5$ & $297.0 \pm 72.5$ & $281.4 \pm 91.1$ & 0.421 \\
\hline \multicolumn{5}{|l|}{ Type of endovascular treatment } \\
\hline Combined intravenous thrombolysis and thrombectomy, n (\%) & $19(26.4)$ & $8(19.0)$ & $11(36.7)$ & 0.094 \\
\hline Intra-arterial thrombolysis, n (\%) & $10(13.9)$ & $9(21.4)$ & $1(3.3)$ & $0.029^{a}$ \\
\hline Direct mechanical thrombectomy, n (\%) & $43(59.7)$ & $25(59.5)$ & $18(60.0)$ & 0.968 \\
\hline Frequency of mechanical thrombectomy, median (IQR) & $2(2-3)$ & $2(1-3)$ & $3(2-3)$ & $0.024^{a}$ \\
\hline Rates of successful recanalization, n (\%) & $62(86.1)$ & 40 (95.2) & $22(73.3)$ & $0.008^{\mathrm{a}}$ \\
\hline \multicolumn{5}{|l|}{ Intracranial haemorrhagic transformation, n (\%) } \\
\hline HI1 & $5(6.9)$ & $4(9.5)$ & $1(3.3)$ & \multirow[t]{4}{*}{0.197} \\
\hline $\mathrm{HI} 2$ & $5(6.9)$ & $2(4.8)$ & $3(10.0)$ & \\
\hline $\mathrm{PH} 1$ & $2(2.8)$ & $0(0.0)$ & $2(6.7)$ & \\
\hline $\mathrm{PH} 2$ & $1(1.4)$ & $0(0.0)$ & $1(3.3)$ & \\
\hline
\end{tabular}

NIHSS National Institutes of Health Stroke Scale, SBP systolic blood pressure, DBP diastolic blood pressure, LDL-C low-density lipoprotein cholesterol, ASPECTS Alberta Stroke Program Early CT Score, $H I$ petechial infarction without space-occupying effect, $P H$ haemorrhage (coagulum) with mass effect ${ }^{\text {a }}$ Statistically significant

SD were lower in patients with a favorable outcome in the successful recanalization group; however no significant difference was observed in the non-successful recanalization group. Lower systolic $\mathrm{SV}, \mathrm{CV}$, and $\mathrm{SD}$ were found in M1 of the MCA lesion group, according to vascular lesions. In the ICA lesion group, the systolic 
Table 2 Baseline characteristics of patients grouped by systolic SV quartile

\begin{tabular}{|c|c|c|c|c|c|}
\hline Variable & $\begin{array}{l}\text { Quartile } 1 \\
(\leq 10.96) \\
n=18\end{array}$ & $\begin{array}{l}\text { Quartile 2 } \\
(10.97-12.71) \\
n=18\end{array}$ & $\begin{array}{l}\text { Quartile 3 } \\
(12.72-14.24) \\
n=18\end{array}$ & $\begin{array}{l}\text { Quartile 4 } \\
(>14.24) \\
n=18\end{array}$ & $P$-value \\
\hline Age (years), mean $\pm S D$ & $64.8 \pm 10.2$ & $60.1 \pm 12.5$ & $64.6 \pm 10.3$ & $69.4 \pm 9.4$ & 0.090 \\
\hline Male, n (\%) & $11(61.1)$ & $12(66.7)$ & $9(50.0)$ & $13(72.2)$ & 0.557 \\
\hline Hypertension, n (\%) & $10(55.6)$ & $12(66.7)$ & $12(66.7)$ & $14(77.8)$ & 0.572 \\
\hline Diabetes mellitus, n (\%) & $5(27.8)$ & $9(50.0)$ & $4(22.2)$ & $5(27.8)$ & 0.287 \\
\hline Coronary heart disease, n (\%) & $3(16.7)$ & $7(38.9)$ & $7(38.9)$ & $4(22.2)$ & 0.330 \\
\hline Atrial fibrillation, $\mathrm{n}(\%)$ & $7(38.9)$ & $6(33.3)$ & $5(27.8)$ & $6(33.3)$ & 0.919 \\
\hline Previous history of cerebrovascular disease, n (\%) & $3(16.7)$ & $3(16.7)$ & $0(0.0)$ & $4(22.2)$ & 0.243 \\
\hline Current smoker, n (\%) & $8(44.4)$ & $12(66.7)$ & $5(27.8)$ & $9(50.0)$ & 0.134 \\
\hline NIHSS score at admission, median (IQR) & $9.5(7.8-14.8)$ & $13(9.0-16.3)$ & $18(8.5-21.3)$ & $16.5(13.8-20.0)$ & 0.070 \\
\hline Glucose level at admission (mg/dL), mean \pm SD & $149.0 \pm 78.1$ & $184.3 \pm 141.3$ & $138.4 \pm 37.2$ & $137.4 \pm 33.7$ & 0.309 \\
\hline $\mathrm{SBP}$ level at admission $(\mathrm{mmHg})$, mean $\pm \mathrm{SD}$ & $143.4 \pm 23.9$ & $156.7 \pm 17.3$ & $158.7 \pm 27.0$ & $156.4 \pm 23.4$ & 0.189 \\
\hline DBP level at admission $(\mathrm{mmHg})$, mean $\pm \mathrm{SD}$ & $81.9 \pm 15.1$ & $87.3 \pm 11.1$ & $82.7 \pm 12.9$ & $88.8 \pm 14.0$ & 0.335 \\
\hline LDL-C at admission (mg/dL), median (IQR) & $43.5(33.9-56.4)$ & $49.0(36.2-56.0)$ & $36.8(30.3-3.56 .7)$ & $46.2(41.5-56.4)$ & 0.384 \\
\hline \multicolumn{6}{|l|}{ TOAST type, n (\%) } \\
\hline Large artery atherosclerosis & $9(50.0)$ & $11(61.1)$ & $12(66.7)$ & $12(66.7)$ & 0.442 \\
\hline Cardioembolism & $6(33.3)$ & $6(33.3)$ & $5(27.8)$ & $6(33.3)$ & 0.377 \\
\hline Clear reason & $3(16.7)$ & $0(0.0)$ & $1(5.6)$ & $0(0.0)$ & \\
\hline Unknown reason & $0(0.0)$ & $1(5.6)$ & $0(0.0)$ & $0(0.0)$ & \\
\hline ASPECTS at admission, median (IQR) & $7.8(8-9)$ & $8(8-9)$ & $7(7-8.3)$ & $7(6-7.3)$ & $<0.001^{\mathrm{a}}$ \\
\hline \multicolumn{6}{|l|}{ Vascular lesion } \\
\hline M1 of the middle cerebral artery & $14(77.8)$ & $16(88.9)$ & $10(55.6)$ & $11(61.1)$ & 0.106 \\
\hline Internal carotid artery & $4(22.2)$ & $2(11.1)$ & $8(44.4)$ & $7(38.9)$ & \\
\hline \multicolumn{6}{|l|}{ Type of anesthesia } \\
\hline Conscious sedation, n (\%) & $17(94.4)$ & $14(77.8)$ & $16(88.9)$ & $16(88.9)$ & 0.491 \\
\hline General anesthesia, n (\%) & $1(5.6)$ & $4(22.2)$ & $2(11.1)$ & $2(11.1)$ & \\
\hline Time from stroke onset to groin puncture $(\mathrm{min})$, mean \pm SD & $284.6 \pm 60.7$ & $300.1 \pm 75.8$ & $308.7 \pm 84.6$ & $268.7 \pm 97.4$ & 0.467 \\
\hline \multicolumn{6}{|l|}{ Type of endovascular treatment } \\
\hline Combined intravenous thrombolysis and thrombectomy, n (\%) & $4(22.2)$ & $7(38.9)$ & $4(22.2)$ & $4(22.2)$ & 0.777 \\
\hline Intra-arterial thrombolysis alone, n (\%) & $4(22.2)$ & $2(11.1)$ & $2(11.1)$ & $2(11.1)$ & \\
\hline Direct mechanical thrombectomy, n (\%) & $10(55.6)$ & $9(50.0)$ & $12(66.7)$ & $12(66.7)$ & \\
\hline Frequency of mechanical thrombectomy, median (IQR) & $1.5(0-3)$ & $1.5(1-2.3)$ & $2(1.8-3)$ & $2.5(2-3.3)$ & $0.038^{\mathrm{a}}$ \\
\hline Rates of successful recanalization, $\mathrm{n}(\%)$ & $18(100.0)$ & $17(94.4)$ & $14(77.8)$ & $13(72.2)$ & $0.048^{\mathrm{a}}$ \\
\hline \multicolumn{6}{|l|}{ Intracranial haemorrhagic transformation, n (\%) } \\
\hline HI1 & $1(5.6)$ & $1(5.6)$ & $1(5.6)$ & $2(11.1)$ & 0.731 \\
\hline HI2 & $2(11.1)$ & $0(0.0)$ & $1(5.6)$ & $2(11.1)$ & \\
\hline $\mathrm{PH} 1$ & $0(0.0)$ & $0(0.0)$ & $1(5.6)$ & $1(5.6)$ & \\
\hline $\mathrm{PH} 2$ & $0(0.0)$ & $0(0.0)$ & $0(0.0)$ & $1(5.6)$ & \\
\hline
\end{tabular}

NIHSS National Institutes of Health Stroke Scale, SBP systolic blood pressure, DBP diastolic blood pressure, LDL-C low-density lipoprotein cholesterol, ASPECTS Alberta Stroke Program Early CT Score, $H I$ petechial infarction without space-occupying effect, $P H$ haemorrhage (coagulum) with mass effect ${ }^{\text {a }}$ Statistically significant

SV was lower amongst those patients with a favorable outcome; other BPV parameters were not found to be different (Table 3).
Influencing factors of 3-month functional independence Table 4 summarizes the univariable and multivariable associations of BP measurements after EVT and other 
A

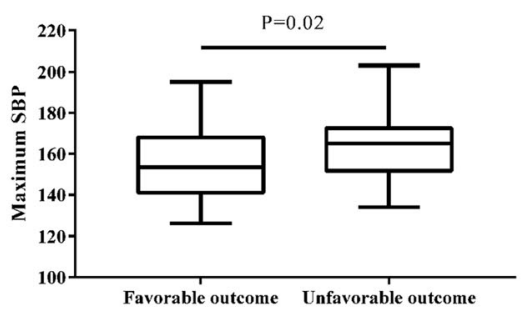

C

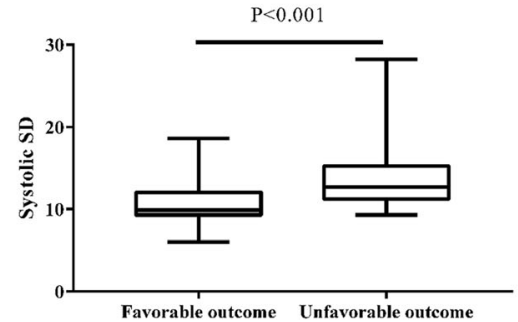

B

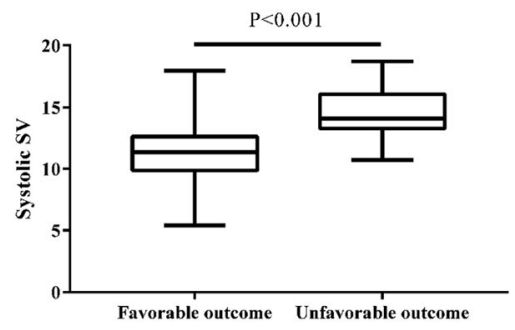

D

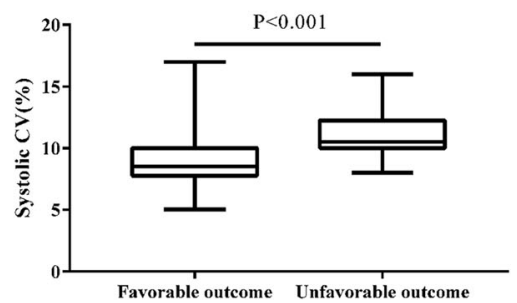

Fig. 1 Maximum SBP (a) and systolic SV (b), SD (c), CV (d) within $24 \mathrm{~h}$ post-EVT in the two groups. EVT endovascular treatment; SBP systolic blood pressure; SV successive variation; SD standard deviation; CV coefficient of variation

clinical characteristics with the 3-month functional prognosis. The following variables were found to be significantly related $(P<0.05)$ to 3 -month functional independence in the initial univariable analysis: NIHSS score at admission, SBP at admission, ASPECTS at admission, M1of the MCA occlusion, frequency of mechanical thrombectomy, measurement of EVT, successful recanalization, maximum $\mathrm{SBP}$ and systolic $\mathrm{SD}, \mathrm{CV}$, and SV post MT. After adjusting for potential confounders, multivariable logistic regression revealed that systolic SV (OR: $4.273,95 \%$ CI: 1.030 to $17.727, P=0.045$ ) was an independent predictor of unfavorable outcome, and a high ASPECTS was independently associated with a better likelihood of a favorable outcome (OR: 0.200, 95\% CI: 0.054 to $0.744, P=0.016$ ).

\section{mRS score distribution according to quartiles of systolic} SV

Patients were divided into 4 groups according to systolic SV values by quartile to clarify the relationship between $\mathrm{SV}$ values and mRS scores (Fig. 2). One-way ANOVA analysis demonstrated that there was a significant in mRS scores at 3 -month of the four groups $(P<0.001)$. In addition, Multiple Post Hoc Comparisons showed compared with the group with high systolic SV, those with lower systolic SV had lower mRS scores at 3 months $(P<0.001, \mathrm{OR}=-1.833,95 \% \mathrm{CI}=-2.722$ to -0.945 for Q1:Q4; $P<0.001, \mathrm{OR}=-1.444,95 \% \mathrm{CI}=-2.333$ to 0.556 for $\mathrm{Q} 1$ :Q3; $P<0.001, \mathrm{OR}=-1.389,95 \% \mathrm{CI}=-$ 2.277 to -0.500 for $\mathrm{Q} 2: \mathrm{Q} 3 ; \mathrm{P}<0.001, \mathrm{OR}=-1.778,95 \%$
$\mathrm{CI}=-2.666$ to -0.889 for $\mathrm{Q} 2: \mathrm{Q} 4 ;$ ), no statistically difference in mRS scores was noted in patients with systolic SV Q1 and Q2 $(P=0.998), \mathrm{Q} 3$ and Q4 $(P=0.659)$. Furthermore, after adjusted for age, sex, frequency of MT, ASPECT score at admission and rates of successful recanalization, the risk of unfavorable outcome at 3-month was significantly decreased in patients with low systolic SV levels compared with the group with high systolic SV levels $(P=0.015, \mathrm{OR}=0.056,95 \% \mathrm{CI}=0.007$ to 0.433 for Q1:Q4). After additional adjustment for hypertension, diabetes mellitus, coronary heart disease, atrial fibrillation, smoking, admission NIHSS scores, admission serum glucose, LDL-C, admission SBP, admission DBP, onset to groin puncture time, vascular lesion and type of EVT, the significance persisted $(P=0.004, \mathrm{OR}=0.008$, $95 \% \mathrm{CI}=0.000$ to 0.141 for $\mathrm{Q} 1: \mathrm{Q} 4)$.

\section{ROC analysis}

ROC analysis demonstrated that the areas under the curve (AUC) of systolic SV for predicting unfavorable outcome was 0.868 (95\% CI: 0.781 to $0.955, P<0.001$; Fig. 3). The optimal cut-off value was 12.499 , which resulted in $93.3 \%$ sensitivity, $73.8 \%$ specificity, $71.1 \%$ PPV, and 91.2\% NPV (Table 5). This indicates that an systolic SV of 12.499 had an excellent predictive value for a poor 3-month functional outcome.

\section{Discussion}

The clinical outcome in patients with ischaemic stroke is affected by many factors, including age, severity of 
Table 3 Comparison of SBP and DBP variability parameters between different subgroups

\begin{tabular}{|c|c|c|c|c|c|c|c|}
\hline \multicolumn{4}{|c|}{ Successful recanalization $(n=62,86.1 \%)$} & \multicolumn{4}{|c|}{ Non-successful recanalization $(n=10,13.9 \%)$} \\
\hline BPV index & Favorable outcome & Unfavorable outcome & $P$ value & BPV index & Favorable outcome & Unfavorable outcome & $P$ value \\
\hline Maximum SBP & $153.4 \pm 15.9$ & $165.4 \pm 16.0$ & 0.006 & Maximum SBP & $172.0 \pm 32.5$ & $158.1 \pm 13.9$ & 0.341 \\
\hline Maximum DBP & $94.8 \pm 9.6$ & $90.4 \pm 6.8$ & 0.063 & Maximum DBP & $106.5 \pm 7.8$ & $94.9 \pm 9.6$ & 0.154 \\
\hline Minimum SBP & $111.2 \pm 14.8$ & $110.0 \pm 13.7$ & 0.751 & Minimum SBP & $131.0 \pm 38.2$ & $103.9 \pm 12.8$ & 0.496 \\
\hline Minimum DBP & $60.0 \pm 10.3$ & $58.3 \pm 9.3$ & 0.498 & Minimum DBP & $64.5 \pm 30.4$ & $59.4 \pm 8.5$ & 0.851 \\
\hline Mean SBP & $130.9 \pm 15.0$ & $134.2 \pm 13.7$ & 0.395 & Mean SBP & $151.7 \pm 33.3$ & $131.1 \pm 18.0$ & 0.239 \\
\hline Mean DBP & $76.1 \pm 8.9$ & $73.7 \pm 8.0$ & 0.296 & Mean DBP & $77.4 \pm 25.5$ & $77.1 \pm 7.5$ & 0.987 \\
\hline Systolic SV & $11.4 \pm 2.3$ & $14.4 \pm 2.0$ & $<0.001$ & Systolic SV & $12.8 \pm 1.8$ & $15.0 \pm 1.9$ & 0.174 \\
\hline Systolic SD & $10.0 \pm 2.4$ & $14.0 \pm 4.3$ & $<0.001$ & Systolic SD & $8.7 \pm 1.6$ & $13.2 \pm 2.6$ & 0.052 \\
\hline Systolic CV & $(8.8 \pm 2.0) \%$ & $(10.8 \pm 1.7) \%$ & $<0.001$ & Systolic CV & $(8.8 \pm 3.1) \%$ & $(11.7 \pm 2.2) \%$ & 0.160 \\
\hline Diastolic SV & $9.8 \pm 3.2$ & $8.8 \pm 2.3$ & 0.182 & Diastolic SV & $12.1 \pm 5.0$ & $10.4 \pm 3.2$ & 0.544 \\
\hline Diastolic SD & $8.3 \pm 2.2$ & $8.0 \pm 2.0$ & 0.610 & Diastolic SD & $8.7 \pm 3.7$ & $8.2 \pm 1.7$ & 0.796 \\
\hline Diastolic CV & $(13.1 \pm 4.8) \%$ & $(12.1 \pm 3.6) \%$ & 0.377 & Diastolic CV & $(17.7 \pm 12.3) \%$ & $(13.8 \pm 5.0) \%$ & 0.459 \\
\hline M1 of MCA & & & & ICA & & & \\
\hline BPV index & Favorable outcome & Unfavorable outcome & $P$ value & BPV index & Favorable outcome & Unfavorable outcome & $P$ value \\
\hline Maximum SBP & $154.2 \pm 16.6$ & $159.5 \pm 14.9$ & 0.278 & Maximum SBP & $154.7 \pm 19.5$ & $168.0 \pm 15.5$ & 0.106 \\
\hline Maximum DBP & $96.6 \pm 8.8$ & $90.8 \pm 8.6$ & 0.032 & Maximum DBP & $89.1 \pm 13.0$ & $92.5 \pm 6.6$ & 0.437 \\
\hline Minimum SBP & $112.9 \pm 16.1$ & $109.1 \pm 15.0$ & 0.420 & Minimum SBP & $108.0 \pm 17.3$ & $107.5 \pm 12.0$ & 0.939 \\
\hline Minimum DBP & $60.0 \pm 11.3$ & $57.6 \pm 8.1$ & 0.450 & Minimum DBP & $61.6 \pm 11.0$ & $59.6 \pm 10.0$ & 0.691 \\
\hline Mean SBP & $132.1 \pm 16.0$ & $133.1 \pm 16.6$ & 0.847 & Mean SBP & $130.9 \pm 18.5$ & $133.8 \pm 12.9$ & 0.678 \\
\hline Mean DBP & $76.1 \pm 9.4$ & $74.4 \pm 7.4$ & 0.512 & Mean DBP & $76.4 \pm 11.0$ & $74.9 \pm 8.8$ & 0.734 \\
\hline Systolic SV & $11.5 \pm 1.9$ & $14.5 \pm 1.9$ & $<0.001$ & Systolic SV & $11.4 \pm 3.9$ & $14.6 \pm 2.1$ & 0.02 \\
\hline Systolic SD & $10.1 \pm 2.0$ & $12.6 \pm 2.6$ & $<0.001$ & Systolic SD & $12.8 \pm 3.3$ & $15.1 \pm 4.7$ & 0.259 \\
\hline Systolic CV & $(8.8 \pm 1.9) \%$ & $(11.0 \pm 2.1) \%$ & $<0.001$ & Systolic CV & $(8.7 \pm 2.5) \%$ & $(11.0 \pm 1.6) \%$ & 0.018 \\
\hline Diastolic SV & $10.4 \pm 3.3$ & $9.8 \pm 2.9$ & 0.533 & Diastolic SV & $7.7 \pm 2.1$ & $8.5 \pm 2.3$ & 0.406 \\
\hline Diastolic SD & $8.5 \pm 2.3$ & $8.3 \pm 1.9$ & 0.709 & Diastolic SD & $7.4 \pm 1.3$ & $7.9 \pm 2.0$ & 0.552 \\
\hline Diastolic CV & $(14.0 \pm 5.3) \%$ & $(13.3 \pm 4.2) \%$ & 0.644 & Diastolic CV & $(10.0 \pm 2.0) \%$ & $(11.6 \pm 3.8) \%$ & 0.288 \\
\hline
\end{tabular}

SBP Systolic blood pressure, DBP Diastolic blood pressure, BPV Blood pressure variability, SD Standard deviation, CV Coefficient of variation, SV Successive variation

stroke, collateral compensation, time of successful reperfusion, and device selected for EVT. BP management and its effect on functional outcome is particularly controversial. A previous study showed that increased systolic BPV positively contributed to symptomatic intracerebral haemorrhage and death after intravenous thrombolysis [16]. However, less is known about the effect of short-term BPV after EVT on the early outcomes of AIS-LVO patients. Our study shows that lower maximum SBP and systolic CV, SV, and SD levels during the first $24 \mathrm{~h}$ after EVT are related to a better 3-month functional outcome, which was consistent with the results reported by Bennett [17].

BPV is divided into physiological and pathological variability, which fluctuates with physiological regulation, environmental changes, and pathological influence. The possible pathophysiological mechanisms regarding short-term BPV in AIS patients with EVT are not clear.
In a previous study, increased BPV may promote shear force of blood vessels and produce vascular inflammation by increasing endothelial expression of cytokines, which affect the structure of the vascular wall and lead to the formation of atherosclerotic plaques [10, 18]. Another hypothesis is that the effects of increased BPV on brain tissue may vary with the degree of impaired cerebral autoregulation [19], and the cerebral blood flow dependent on cerebral perfusion pressure and blood viscosity [20]. Endovascular therapy can not only stimulate endothelial cells, but also change the cerebral perfusion pressure and vascular resistance in LVO-AIS patients. Therefore, there is a potential correlation between blood pressure variability and outcome in LVOAIS patients after intra-arterial treatment.

BPV are commonly quantified by calculating SD, CV, and SV [10]. Several studies have shown that higher systolic CV or SD is associated with poor prognosis after 
Table 4 Univariate and multivariate analysis of the favorable outcomes after EVT

\begin{tabular}{|c|c|c|c|c|}
\hline \multirow[t]{2}{*}{ Variable } & \multicolumn{2}{|l|}{ Univariable logistic regression analysis } & \multicolumn{2}{|c|}{ Multivariable logistic regression analysis } \\
\hline & $\overline{\mathrm{OR}}(95 \% \mathrm{Cl})$ & $P$ value $^{*}$ & $\overline{\mathrm{OR}}(95 \% \mathrm{Cl})$ & $P$ value \\
\hline Age & $1.005(0.963-1.050)$ & 0.817 & & \\
\hline Male & $1.200(0.457-3.151)$ & 0.711 & & \\
\hline Hypertension & $1.000(0.370-2.702)$ & 1.000 & & \\
\hline Coronary heart disease & $1.071(0.383-2.997)$ & 0.895 & & \\
\hline Atrial fibrillation & $0.591(0.213-1.641)$ & 0.313 & & \\
\hline Diabetes mellitus & $0.368(0.124-1.089)$ & 0.071 & & \\
\hline Smoking & $0.765(0.298-1.962)$ & 0.577 & & \\
\hline Glucose level at admission & $0.893(0.755-1.057)$ & 0.189 & & \\
\hline NIHSS at admission & $1.072(1.002-1.148)$ & 0.045 & $0.931(0.808-1.073)$ & 0.325 \\
\hline SBP level at admission & $1.036(1.010-1.063)$ & 0.006 & $1.045(0.993-1.100)$ & 0.092 \\
\hline DBP level at admission & $1.025(0.988-1.063)$ & 0.189 & & \\
\hline LDL-C at admission & $1.170(0.724-1.891)$ & 0.521 & & \\
\hline Conscious sedation & $1.900(0.465-7.769)$ & 0.372 & & \\
\hline ASPECTS at admission & $0.268(0.138-0.522)$ & $<0.001$ & $0.200(0.054-0.744)$ & 0.016 \\
\hline Time from stroke onset to groin puncture & $0.998(0.992-1.003)$ & 0.415 & & \\
\hline M1 of the MCA occlusion & $0.229(0.077-0.675)$ & 0.008 & $0.076(0.005-1.078)$ & 0.057 \\
\hline Frequency of mechanical thrombectomy & $0.098(0.011-0.860)$ & 0.036 & $1.499(0.038-59.877)$ & 0.830 \\
\hline Combined intravenous thrombolysis and thrombectomy & $2.461(0.844-7.172)$ & 0.099 & & \\
\hline Intra-arterial thrombolysis & $0.097(0.012-0.801)$ & 0.030 & $0.012(0.000-1.457)$ & 0.071 \\
\hline Successful recanalization & $0.138(0.027-0.075)$ & 0.017 & $0.030(0.001-1.842)$ & 0.095 \\
\hline Maximum SBP post EVT ${ }^{a}$ & $1.036(1.004-1.069)$ & 0.803 & $0.894(0.777-1.028)$ & 0.116 \\
\hline Maximum DBP post EVT ${ }^{\mathrm{a}}$ & $0.953(0.901-1.008)$ & 0.091 & & \\
\hline Minimum SBP post EVT ${ }^{\mathrm{a}}$ & $0.983(0.952-1.015)$ & 0.297 & & \\
\hline Minimum DBP post $\mathrm{EVT}^{\mathrm{a}}$ & $0.983(0.939-1.030)$ & 0.482 & & \\
\hline Mean SBP post EVT ${ }^{a}$ & $1.006(0.976-1.037)$ & 0.686 & & \\
\hline Mean DBP post EVT $T^{a}$ & $0.980(0.929-1.034)$ & 0.456 & & \\
\hline Systolic SD post EVT ${ }^{a}$ & $1.531(1.203-1.948)$ & 0.001 & $1.217(0.803-1.842)$ & 0.355 \\
\hline Systolic CV post EVT ${ }^{\mathrm{a}}$ & $2.732 \mathrm{E}+28(8.024 \mathrm{E}+12-9.303 \mathrm{E}+43)$ & $<0.001$ & $0.000(0.000-7.704 \mathrm{E}+24)$ & 0.221 \\
\hline Systolic SV post EVT ${ }^{a}$ & $2.046(1.444-2.898)$ & $<0.001$ & $4.273(1.030-17.727)$ & 0.045 \\
\hline
\end{tabular}

stroke [21, 22]; however, systolic SV, an indicator of systolic blood pressure variability, is more commonly used in many studies, because it can better reflect the time-series variability of $\mathrm{BP}$, while other parameters, such as SD and $\mathrm{CV}$, ignore the temporal change of data, resulting in the same $\mathrm{SD}$ or $\mathrm{CV}$ in individuals with different clinical characteristics [23]. In our study, we confirmed that the systolic SV, rather than systolic CV and SD, was closely associated with 3-month functional outcome. Lower systolic SV levels may be beneficial to achieving 3-month functional independence. After adjusting for various confounders, the correlation remained significant. These findings suggest that maintaining a stable BP may be more useful than merely controlling the BP levels after EVT.

A study of 217 patients who underwent MT showed that a higher maximum SBP was closely related to 3month mortality and poor outcome. Each $10 \mathrm{mmHg}$ increase in maximum SBP during the first $24 \mathrm{~h}$ post MT was associated with a lower 3-month functional prognosis and a higher odds of 3-month mortality [24]. Although our study did not find the maximum SBP to be an influential factor for functional outcome, this may be related to our study subjects' characteristics. Our 


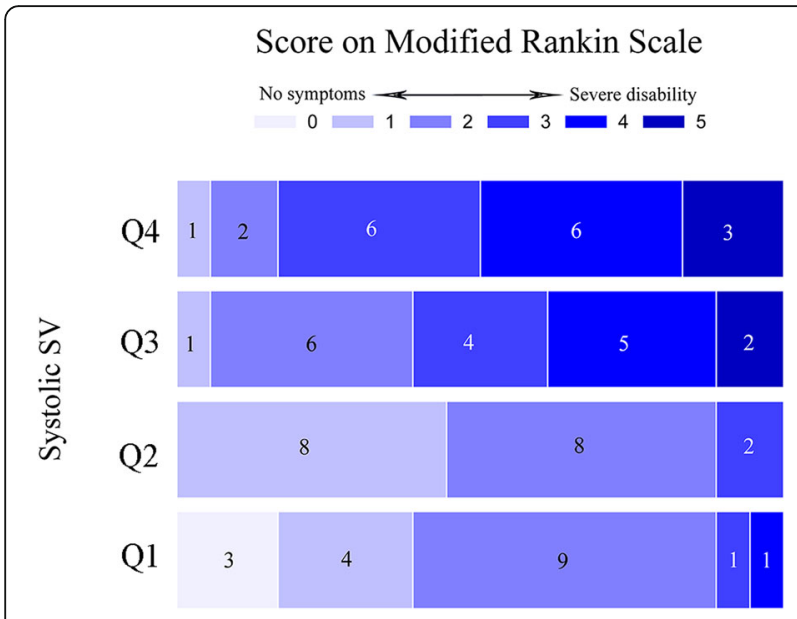

Fig. 2 Modified Rankin Scale scores distribution according to quartiles of systolic SV

research found that the rate of successful recanalization was higher in the favorable outcome group, which also had lower maximum SBP, indirectly suggesting that patients with successful reperfusion are more likely to benefit from lower SBP [25].

Some studies showed that BP within the first $48 \mathrm{~h}$ after a stroke showed a U-shaped correlation with clinical outcome [17, 26], particularly in patients with non-recanalization. The authors argued that patients with unsuccessful recanalization had larger infarct size and ischaemic penumbra, and impaired cerebral autoregulation led to further enlargement of the ischaemic

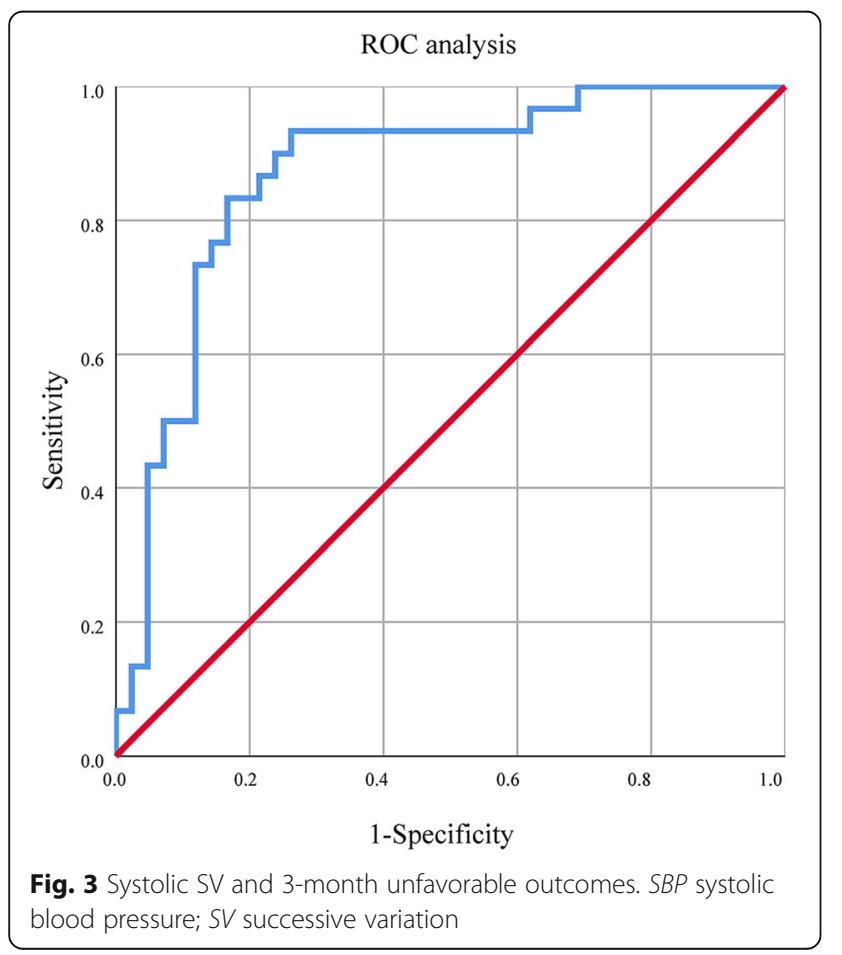

penumbra [17]. The effect of BPV on the ischaemic penumbra is different from that on the infarct core [10]. In the hours after onset, potentially ischaemic penumbra are particularly sensitive to blood pressure fluctuations, with sudden drops in blood pressure increasing the risk of tissue ischaemia and reducing the chance of reperfusion, and sudden increases in blood pressure increasing the risk of bleeding. Patients who received EVT and had unsuccessful reperfusion enlarged the ischaemic penumbra, which was sensitive to blood pressure variability, greatly increasing the risk of neurological deterioration caused by BPV. In addition, cerebral ischaemia and MT itself may lead to the destruction of blood-brain barrier, resulting in vasogenic edema and haemorrhagic transformation after infarction. Moreover, iatrogenic injury to endothelial cells during MT can cause a series of reperfusion-related injuries [27] that not only increase intracranial haemorrhage associated with SBP, but also lead to adverse functional prognosis. In this study, the subjects with low systolic SV had a higher rate of successful recanalization, we therefore deduced that successful recanalization is a significant factor for systolic SV. In addition, owing to the small number of cases of patients without recanalization, no significant difference was found between the two groups in terms of functional outcome. Future studies should focus on enlarging the sample size to be more adequately powered for these sorts of subanalyses. Another study also showed that the peak level of SBP was closely related to poor outcome regardless of whether LVO recanalization was achieved or not. The authors suggested that this is probably because abnormally elevated BP may be associated with potential collateral circulation damage [28].

The impairment of cerebral autoregulation is influenced by infarct size [29]. Thus, BPV may exert a greater pathophysiological role in patients with severe stroke than those with mild stroke. In our study, patients with favorable prognosis were more likely to have M1 of the middle cerebral artery affected, which may, in theory, produce a smaller infarct volume. Subgroup analysis in our cohort also confirmed that those with M1 of MCA lesions, the systolic SV, CV, and SD were lower in the favorable outcome group.

Several limitations of the present study need to be acknowledged. First, this was a single-centre retrospective study with a relatively small sample size. Thus, selection bias in baseline data was unavoidable. Second, a recent study demonstrated that BPV post MT may increase the rate of symptomatic intracranial haemorrhage $(\mathrm{sICH})$ [10], but we did not evaluate the relationship between BPV and $\mathrm{sICH}$ because the patients who developed intracranial haemorrhage during follow-up were classified according to ECASS criteria without the clinical classification for sICH. Third, variable reasons such as 
Table 5 Cut-off values of systolic SV

\begin{tabular}{lccccc}
\hline Values & Best cut-off & Sensitivity (\%) & Specificity (\%) & PPV $^{*}(\%)$ & NPV $^{*}(\%)$ \\
\hline Systolic SV & 12.499 & 93.3 & 73.8 & 71.1 & 91.2 \\
\hline SV Successive variation. PPV Positive predictive values: NPV Negative predictive values & &
\end{tabular}

SV Successive variation; PPV Positive predictive values; NPV Negative predictive values

the varying time from stroke onset to arrival at our hospital for the first BP measurement and differences in time intervals between BP measurements may have introduced bias in our results. However, we exerted every effort to provide reliable dates to mitigate the inherent limitations. Fourth, SD, CV and SV are not suitable for the long-term evaluation of BPV after EVT; severe stroke with a poor prognosis may give rise to greater variability in BP. Therefore, additional well-designed and larger prospective randomized cohort studies are required to confirm the association of BPV and functional prognosis and to determine optimal strategies to reduce the BPV.

\section{Conclusions}

Decreased systolic SV following intra-arterial therapies result in favorable 3-month outcomes. Systolic SV may therefore be a novel predictor of functional prognosis in LVO patients.

\section{Supplementary information}

Supplementary information accompanies this paper at https://doi.org/10. 1186/s12883-019-1457-5.

Additional file 1:Table S1. Intracerebral haemorrhage of patients postEVT. (16 kb)

\section{Abbreviations}

AIS: Acute ischaemic stroke; ASPECTS: Alberta Stroke Program Early CT Score; AUC: Area under the curve; BP: Blood pressure; BPV: Blood pressure variability; Cl: Confidence interval; CV: Coefficient of variation; DBP: Diastolic blood pressure; ECASS: European Cooperative Acute Stroke Study; EVT: Endovascular treatment; HI: Petechial infarction without spaceoccupying effect; IQR: Interquartile range; LDL-C: Low-density lipoprotein cholesterol; LVO: Large-vessel occlusion; MCA: middle cerebral artery; mRS: Modified Rankin Scale; MT: Mechanical thrombectomy; NIHSS: National Institutes of Health Stroke Scale; OR: Odds ratio; PH: Haemorrhage (coagulum) with mass effect; ROC: Receiver operating characteristic SBP: Systolic blood pressure; SD: Standard deviation; sICH: Symptomatic intracerebral haemorrhage; SV: Successive variation; TICl: Thrombolysis in Cerebral Infarction; TOAST: Trial of ORG 10172 in acute stroke treatment

\section{Acknowledgements}

Not applicable.

\section{Authors' contributions}

WRL conceived the study and revised the manuscript. TLZ wrote the manuscript and analyzed the data. XLW, CW, FZ, and SWG performed intraarterial treatment and collected the data and interpreted the analysis. SQL, XDZ and JS critically revised the manuscript for important intellectual content. All authors read and approved the final manuscript.

\section{Funding}

This work was supported by ShanXi Science and Technology Department (CN): Establishment of Information Service for Stroke Treatment in Taiyuan Central Hospital (Grant No. 2015091012). The funding bodies did not influence the study design, the collection, analysis, and interpretation of data, the manuscript writing, and the decision to submit it for publication.

Availability of data and materials

The datasets used and/or analysed during the current study are available from the corresponding author on reasonable request.

\section{Ethics approval and consent to participate}

This study was approved by the ethic committee of the Taiyuan Central Hospital, ShanXi, China. Patient's consents were waived by the ethic committee of the ethic committee of the Taiyuan Central Hospital, due to the retrospective design of the study.

\section{Consent for publication}

Not applicable.

\section{Competing interests}

The authors declare that they have no competing interests.

Received: 17 April 2019 Accepted: 4 September 2019

Published online: 26 September 2019

\section{References}

1. Saver JL, Goyal M, Bonafe A, et al. Stent-retriever thrombectomy after intravenous t-PA vs. t-PA alone in stroke. N Engl J Med. 2015;372:2285-95.

2. Goyal M, Demchuk AM, Menon BK, et al. Randomized assessment of rapid endovascular treatment of ischemic stroke. N Engl J Med. 2015;372:1019-30.

3. Campbell BC, Mitchell PJ, Kleinig TJ, et al. Endovascular therapy for ischemic stroke with perfusion-imaging selection. N Engl J Med. 2015;372:1009-18.

4. Berkhemer OA, Fransen PS, Beumer D, et al. A randomized trial of intraarterial treatment for acute ischemic stroke. N Engl J Med. 2015; 372:11-20.

5. Jovin TG, Chamorro A, Cobo E, et al. Thrombectomy within 8 hours after symptom onset in ischemic stroke. N Engl J Med. 2015;372:2296-306.

6. Bracard S, Ducrocq X, Mas JL, et al. Mechanical thrombectomy after intravenous alteplase versus alteplase alone after stroke (THRACE): a randomised controlled trial. Lancet Neurol. 2016;15:1138-47.

7. Powers WJ, Rabinstein AA, Ackerson T, et al. 2018 guidelines for the early Management of Patients with Acute Ischemic Stroke: a guideline for healthcare professionals from the American Heart Association/American Stroke Association. Stroke. 2018:49:e46-e110.

8. Chinese Sociaty of Neurology, Chinese Stroke Society, Neurovascular Intervention Group of Chinese Society of Neurology. Chinese guidelines for the endovascular treatment of acute ischemic stroke 2018. Chin J Neurol. 2018;51:683-91.

9. Dawson SL, Panerai RB, Potter JF. Serial changes in static and dynamic cerebral autoregulation after acute ischaemic stroke. Cerebrovasc Dis. 2003;16:69-75.

10. Manning LS, Rothwell PM, Potter JF, et al. Prognostic significance of shortterm blood pressure variability in acute stroke: systematic review. Stroke. 2015:46:2482-90.

11. Chinese Sociaty of Neurology, Chinese Stroke Society. Chinese guidelines for diagnosis and treatment of acute ischemic stroke 2014. Chin J Neurol. 2015:48:246-57.

12. Chinese Sociaty of Neurology, Chinese Stroke Society, Neurovascular Intervention Group of Chinese Society of Neurology. Chinese guidelines for the endovascular treatment of acute ischemic stroke 2015. Chin J Neurol. 2015:48:356-61.

13. Higashida RT, Furlan AJ, Roberts $H$, et al. Trial design and reporting standards for intra-arterial cerebral thrombolysis for acute ischemic stroke. Stroke. 2003;34:e109-37.

14. Yaghi S, Willey JZ, Cucchiara B, et al. Treatment and outcome of hemorrhagic transformation after intravenous Alteplase in acute ischemic stroke: a scientific statement for healthcare professionals from the American Heart Association/American Stroke Association. Stroke. 2017;48:e343-61. 
15. Endo K, Kario K, Koga M, et al. Impact of early blood pressure variability on stroke outcomes after thrombolysis: the SAMURAI rt-PA registry. Stroke. 2013:44:816-8.

16. De Havenon A, Bennett A, Stoddard GJ, et al. Determinants of the impact of blood pressure variability on neurological outcome after acute ischaemic stroke. Stroke Vasc Neurol. 2017;2:1-6.

17. Bennett AE, Wilder MJ, McNally JS, et al. Increased blood pressure variability after endovascular thrombectomy for acute stroke is associated with worse clinical outcome. J Neurointerv Surg. 2018;10:823-7.

18. Kolyviras A, Manios E, Georgiopoulos G, et al. Differential associations of systolic and diastolic time rate of blood pressure variation with carotid atherosclerosis and plaque echogenicity. J Clin Hypertens (Greenwich). 2017;19(11):1070-7.

19. Ko Y, Park JH, Yang MH, et al. The significance of blood pressure variability for the development of hemorrhagic transformation in acute ischemic stroke. Stroke. 2010;41(11):2512-8.

20. Dankbaar JW, Slooter AJ, Rinkel GJ, Schaaf IC. Effect of different components of triple-H therapy on cerebral perfusion in patients with aneurysmal subarachnoid haemorrhage: a systematic review. Crit Care. 2010;14(1):R23.

21. Sare GM, Ali M, Shuaib A, Bath PM. Relationship between hyperacute blood pressure and outcome after ischemic stroke: data from the VISTA collaboration. Stroke. 2009:40(6):2098-103.

22. Geeganage C, Tracy M, England T, et al. Relationship between baseline blood pressure parameters (including mean pressure, pulse pressure, and variability) and early outcome after stroke: data from the Tinzaparin in acute Ischaemic stroke trial (TAIST). Stroke. 2011:42(2):491-3.

23. Xu B, Ji Q, Zhang Y, Shen L, Cao M, Cai K. Postoperative blood pressure variability exerts an influence on clinical outcome after coil embolization of ruptured intracranial aneurysms. Neurol Res. 2017;39(9):813-8.

24. Goyal N, Tsivgoulis G, Pandhi A, et al. Blood pressure levels post mechanical thrombectomy and outcomes in large vessel occlusion strokes. Neurology. 2017:89:540-7.

25. Maier IL, Tsogkas I, Behme D, et al. High systolic blood pressure after successful endovascular treatment affects early functional outcome in acute ischemic stroke. Cerebrovasc Dis. 2018:45:18-25.

26. Leonardi-Bee J, Bath PM, Phillips SJ, et al. Blood pressure and clinical outcomes in the international stroke trial. Stroke. 2002;33:1315-20.

27. Teng D, Pannell JS, Rennert RC, et al. Endothelial trauma from mechanical thrombectomy in acute stroke: in vitro live-cell platform with animal validation. Stroke. 2015;46:1099-106

28. Goyal N, Tsivgoulis G, Pandhi A, et al. Blood pressure levels post mechanical thrombectomy and outcomes in non-recanalized large vessel occlusion patients. J Neurointerv Surg. 2018;10:925-31.

29. Reinhard M, Rutsch S, Lambeck J, et al. Dynamic cerebral autoregulation associates with infarct size and outcome after ischemic stroke. Acta Neurol Scand. 2012;125(3):156-62.

\section{Publisher's Note}

Springer Nature remains neutral with regard to jurisdictional claims in published maps and institutional affiliations.

Ready to submit your research? Choose BMC and benefit from:

- fast, convenient online submission

- thorough peer review by experienced researchers in your field

- rapid publication on acceptance

- support for research data, including large and complex data types

- gold Open Access which fosters wider collaboration and increased citations

- maximum visibility for your research: over $100 \mathrm{M}$ website views per year

At $\mathrm{BMC}$, research is always in progress.

Learn more biomedcentral.com/submissions 